\title{
Apical Hypertrophic Cardiomyopathy and Myocardial Infarction in a Young Adult, a Rare Combination
}

\author{
GAUTAM DATTA, ${ }^{1}$ DIPANKAR MUKHERJEE, ${ }^{2}$ BHUBANMAJHI DM,${ }^{3}$ RUPESH SINGH DM, ${ }^{4}$ SUDHIR BHATTACHARYA 5
}

\begin{abstract}
A teenage boy of 19 years without traditional risk factors for coronary artery disease, presented with typical anginal pain with ST-T changes in anterior leads and elevated cardiac troponin T. Investigation revealed the patient having apical hypertrophic cardiomyopathy with normal coronary angiography and a spade shaped apex on LV angiography. With a diagnosis of microvascular infraction, further investigations revealed elevated LP (a), serum homocysteine and low HDL probably playing a contributory role.
\end{abstract}

Keywords: Apical hypertrophic cardiomyopathy, Microvascular infarction, homocysteine,LP (a)]

\section{Introduction}

Hypertrophic Cardiomyopathy is one of the commonest genetic cardiac abnormalities involving cardiac sarcomeres. Among the different phenotypic variants, apical hypertrophic cardiomyopathy is usually considered to be benign. Coronary microvascular abnormalities have been reported in such cases though frank infarct is quite rare. Here we have reported a teenage boy of apical hypertrophic cardiomyopathy with microvascular infarction associated with elevated LP (a) and serum homocysteine.

\section{Case History}

A 19 year aged male student without any history of competitive athletic activities, presented with severe compressing chest pain at rest and radiating to the left arm for previous four hours. He is non-diabetic.non-hypertensive and without any history of addiction or premature CAD in the family. Physical examination revealed pulse rate of 95/ $\mathrm{min}, \mathrm{BP}-118 / 78 \mathrm{mmHg}$ and normal auscultatory findings. A 12 lead ECG revealed symmetrical $\mathrm{T}$ wave inversion in Lead I, II, III, AVL, AVF and lead V4-V6 (Fig 1). Subsequently quantitative troponin $\mathrm{T}$ estimation was found to be positive and $1 \mathrm{ng} / \mathrm{ml}$. CK and CK-MB level was 90IU(25-195) and 12 IU(0-24) respectively. Echocardiographic evaluation however did not show any regional wall motion abnormalities and patient had moderate left ventricular hypertrophy involving all the walls and particularly the apex with apical obliteration

1. Associate Prof., Burdwan Medical College

2. Associate Prof, ICVS, IPGME\& R, Kolkata

3. ICVS, IPGME\& R, Kolkata

4. ICVS, IPGME\& R, Kolkata

5. Medical Officer, ICVS, IPGME\& R, Kolkata

Correspondence: Assoc. Prof. Dr. Gautam Datta, (MD, DNB, DM), P-3, Lake Garden,48/4,Sultan Alam Road,Kolkata-700033, Mobile: 09433415086, e-mail Goutamdattadn@yahoo.in

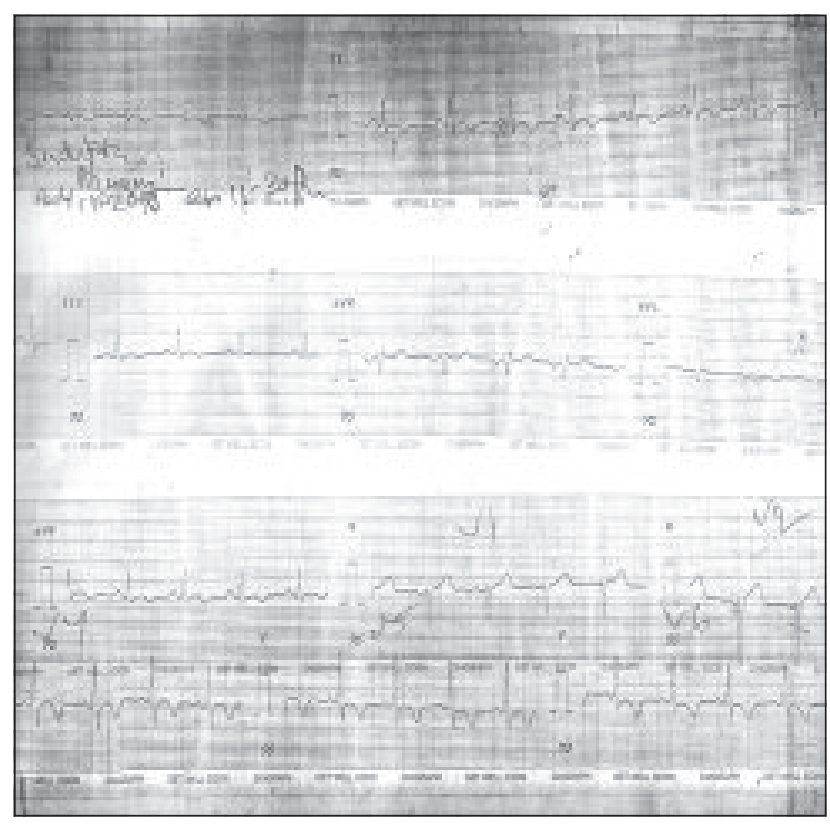

Fig.-1: ECG showing marked $T$ wave abnormalities in inferior and lateral leads.

(Fig 2). Septal and posterior wall diameter was $14 \mathrm{~mm}$ each whereas the apex was $17 \mathrm{~mm}$ in diameter. There was no SAM (systolic anterior motion of mitral valve leaflet), significant LV outflow or mid cavity gradient. His Hemogram, fasting blood glucose, blood urea, creatinine and electrolytes were normal. Lipid profile showed Total cholesterol- $152 \mathrm{mg} / \mathrm{dl}$, LDL- 108, VLDL-29, TG-145 and HDL was $25 \mathrm{mg} / \mathrm{dl}$. Lipoprotein (a) was $50.7 \mathrm{mg} / \mathrm{dl}$ (normal $-<30 \mathrm{mg} / \mathrm{dl}$ ) and serum homocysteine level $20.54 \mathrm{micromol} / \mathrm{L}(3.7-13.9)$ were high. Subsequently coronary angiogram and LV angiogram was performed that showed normal epicardial coronary arteries and a spade shaped LV angiogram with apical obliteration (Fig 3,4,5). In view of the positive myocardial biomarkers, ECG changes and symptoms a diagnosis of non ST segment MI was made and patient was put on treatment accordingly 
with excellent recovery. However normal epicardial coronaries, ECG changes and echocardiographic and LV angiographic findings suggested diagnosis of microvascular myocardial infraction and apical hypertrophic cardiomyopathy in this case.

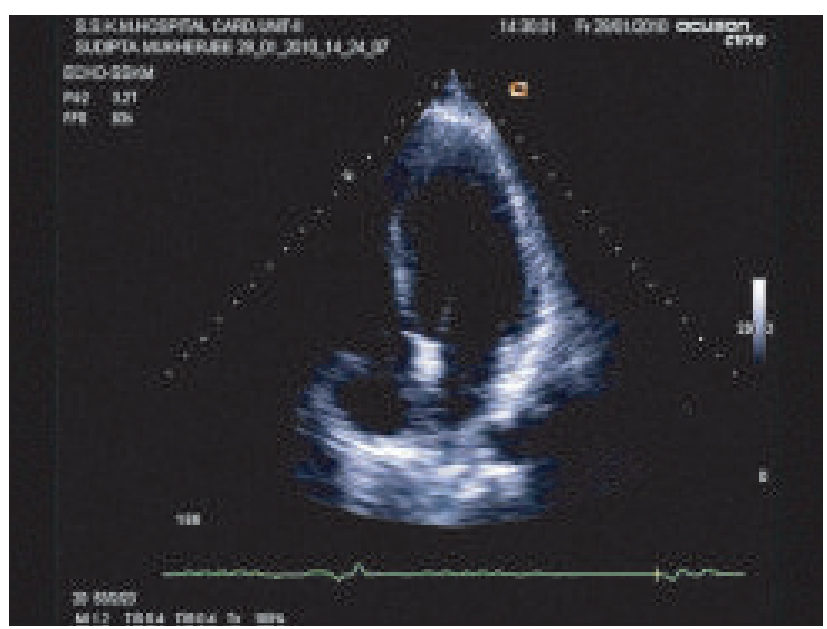

Fig.-2: Apical four chamber view showing apical hypertrophy

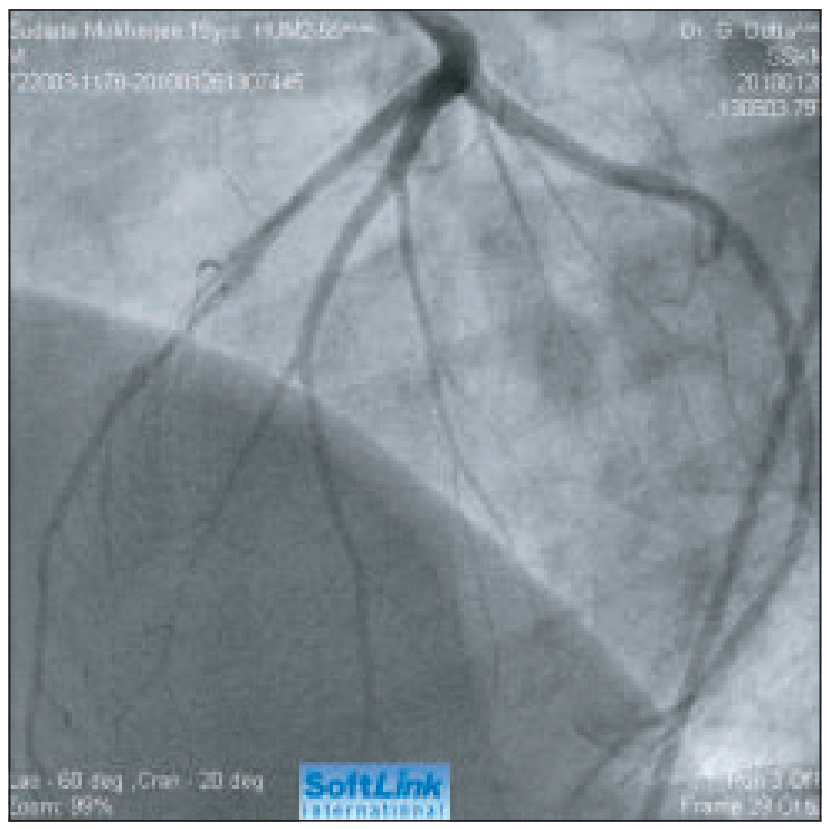

Fig.-3: Coronary angiogram showing normal LAD \& LCX

\section{Discussion}

Hypertrophic Cardiomyopathy is the most common genetic cardiovascular diseases caused by a multitude of mutatations affecting genes encoding cardiac sarcomeres. ${ }^{1}$ Apical HCM is a variant with predominant involvement of the apex of the heart, was first described in Japanese males in $1976,{ }^{2}$ and estimated to represent $25 \%$ of Japanese patients with HCM. Segmental wall thickening mostly confined to the distal

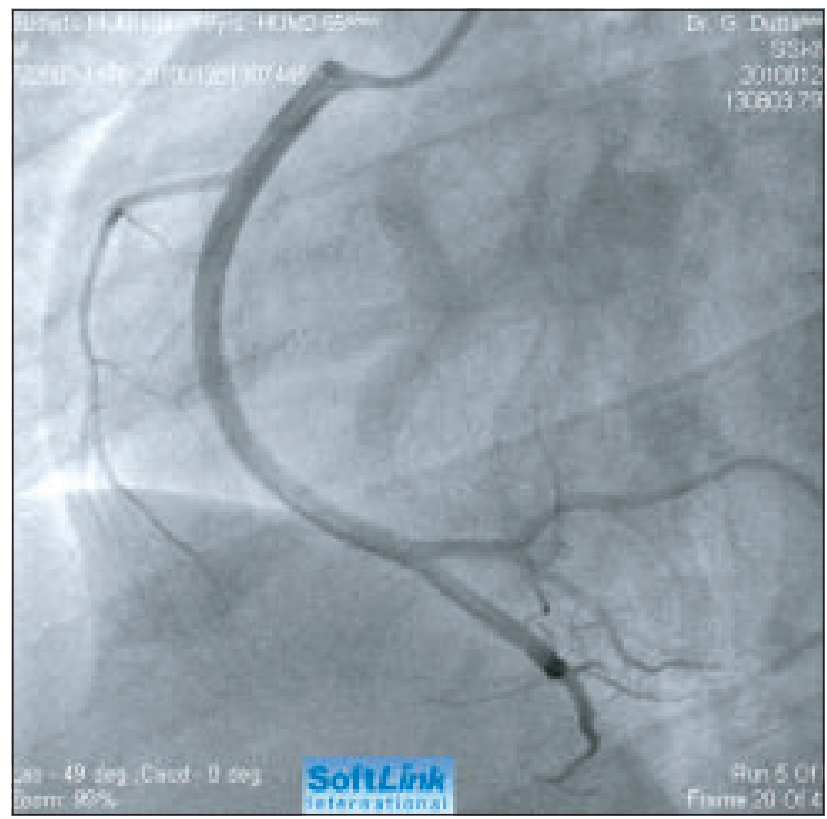

Fig.-4: Coronary angiogram showing normal RCA

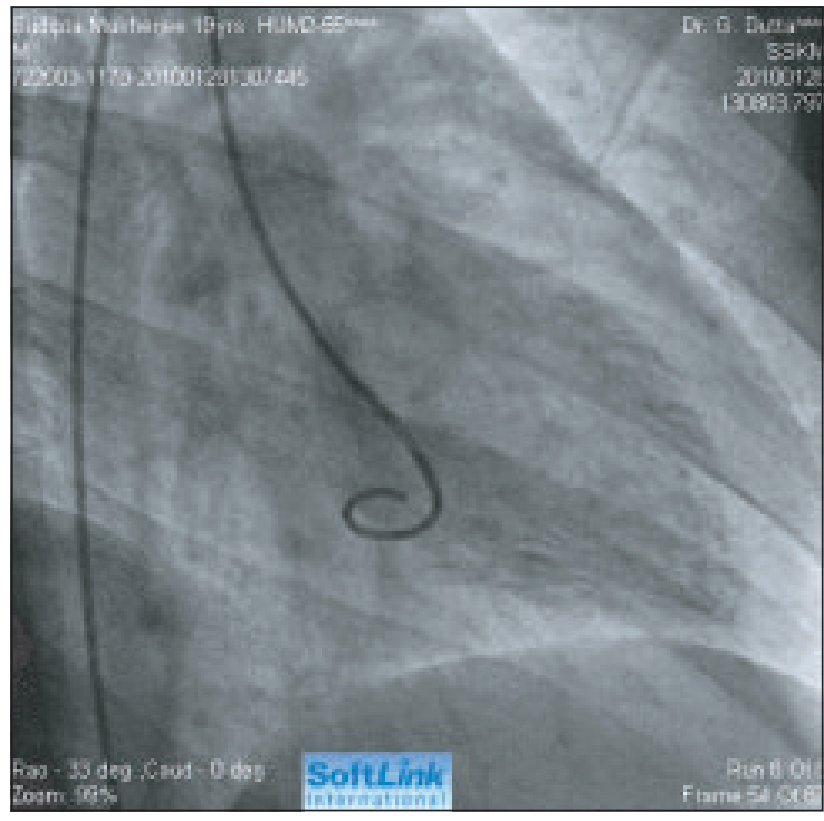

Fig.-5: LV angiogram showing spade shape $L V$

portion of $\mathrm{LV}$ chamber, with marked $\mathrm{T}$ wave inversion on the electrocardiogram are distinctive features of apical HCM. Genetic relation between typical HCM and apical HCM is incompletely understood. ${ }^{3}$

Regional myocardial ischemia in the absence of coronary artery disease is not uncommon in HCM as demonstrated by stress ECG, myocardial perfusion imaging etc. Microvascular dysfunction represents a predisposing factor 
for myocardial ischaemia which is a common feature of hypertrophic cardiomyopathy.It is a strong independent predictor of clinical deterioration and death.Severe microvascular dysfunction is often present in patients with no symptoms and may precede clinical deterioration by years. ${ }^{4}$ Severe impairment of coronary vasodilator response to dypiridamole infusion in PET scan is strongly associated with long term adverse LV remodelling and systolic dysfunction in HCM patients. ${ }^{5}$ In hypertrophic cardiomyopathy abnormal intramural coronary arteries are characterized by thickening of vessel wall due to proliferation of medial and intimal components particularly smooth muscle cells and collagen. They may be located in ventricular septum,anterior left ventricular free wall or posterior free wall. ${ }^{6}$ Potential cause ofmicrovascularangina may include microvascular abnormalities and inadequate capillary densities in comparison to increased LV mass. Abnormal intramural coronaries with increased intimal and medial thickening and myocardial bridging, septal perforator artery compression, coronary artery spasm, elevated filling pressures related to impaired diastolic relaxation and increased oxygen demand due to dynamic LV outflow gradients are also potential mechanism of myocardial ischemia. $^{7}$

In general, patients with apical HCM have a benign course. Symptoms of angina and exertionaldyspnea are well controlled with agents like beta blockers and calcium channel blockers. About half of the patients remain asymptomatic on long term. There have been reports of myocardial infarction, atrial fibrillation or stroke in about one third patients. ${ }^{8}$ KyungTae Jung et al reported a case of apical hypertrophic cardiomyopathy with multiple coronary artery thrombosis. ${ }^{9}$

Homocysteine is a risk factor for cardiovascular disease but treatment with B vitamins didn't lower risk of recurrent cardiovascular disease rather it was harmful ${ }^{10}$.Kamstrup et al conducted Mendelian randomization study to assess LP(a) and myocardial infarction and found that LP(a) gene KIR-2 variants are inversely associated with risk of myocardial infarction.

However combination of apical hypertrophic cardiomyopathy with normal coronaries and microvascular infarction in such a young individual has not been reported. Moreover presence of traditional risk factors of south Asian like low HDL, increased LP (a) and increased homocysteine level in our case is unique and might have contributed to microvascular abnormalities in addtition to the microvascular defect inherent to hypertrophic cardiomyopathies.

\section{Conclusion}

Hypertrophic cardiomyopathy, even its apical variant is not a completely benign disease, and microvascular dysfunction is a well recognized entity. In combination with pre-existing risk factors, acute coronary events may occur even in young individuals where such possibilities are thought to be extremely rare. Aggressive management of risk factors are needed in such individuals to avoid coronary events.

\section{Conflict of Interest : None}

\section{References}

1. Maron BJ, TowbinJA, Thiene G, et al: Contemporary definition and classification of the cardiomyopathies. An American Heart Association Scientific Statement. Circulation 2006; 113:1807,

2. Sakamoto T, Tei C, Murayama M, Ichiyasu H, Hada Y. Giant Twave inversion as a manifestation of asymmetrical apical hypertrophy(AAH) of the left ventricle. Echocardiographic and ultrasonocardiotomographic study. Jpn Heart J. 1976 Sep; 17(5):611-629.

3. Arad M, Penas-Lado M, Monserrat L, Maron BJ, Sherrid M, HoCY,Barr S, Karim A, Olson TM, Kamisago M, Seidman JG, SeidmanCE.Gene mutations in apical hypertrophic cardiomyopathy. Circulation 2005;112:2805-11.

4. Franco Cecchi, IacopoOlivotto, Roberto Gistri, Roberto Lorenzoni, GiampaoloChiriatti, and Paolo G. Camici, Coronary Microvascular Dysfunction and Prognosis in Hypertrophic Cardiomyopathy; NEJM 2003; 349: 1027-1035

5. Olivotto I, Cecchi F, Gistri R, Lorenzoni R, Chiriatti G, Girolami F, Torricelli F, Camici PG: Relevance of coronary microvascular flow impairment to long-term remodeling and systolic dysfunction in hypertrophic cardiomyopathy.J Am CollCardiol. 2006 Mar 7; 47(5):1043-8.

6. Maron BJ, WolfsonJK, Epstein SE, Roberts WC:Intramural ("small vessel") coronary artery disease in hypertrophic cardiomyopathy.J Am CollCardiol. 1986 Sep; 8(3):545-57.

7. MaronBJ, et al; ACC/ESC clinical expert consensus document on hypertrophiccardiomyopathy. A report of the American college of cardiology foundation task force on clinical expert consensus documents and the European society of cardiology committee for practice guidelines.JAmCollCardiol 2003; 42:1687-713

8. Errikson J, Sonneberg B, Woo Anna et al. Long-term outcome in patients with apical hypertrophic cardiomyopathy: J Am CollCardiol, 2002; 39:638-645

9. Kyung-Tae Jung, Soon-Chang Park, , Yoo-Jung Choi, ,et al: A Case of Apical Hypertrophic Cardiomyopathy Combined Acute Myocardial Infarction with Multiple Coronary Thrombosis; J Cardiovasc Ultrasound 2008;16(3):96-98.

10. BønaaKH, Njølstad I, Ueland PM, Schirmer H, Tverdal A, Steigen T, Wang H, Nordrehaug JE, Arnesen E, Rasmussen $\mathrm{K}$;Homocysteine lowering and cardiovascular events after acute myocardial infarction.) N Engl J Med. 2006 Apr 13; 354(15): 1578-88 